PROCEEDINGS OF THE

AMERICAN MATHEMATICAL SOCIETY

Volume 128 , Number 11, Pages 3177-3183

S 0002-9939(00)05537-4

Article electronically published on June 6, 2000

\title{
GROUPS ACTING ON QUASICONVEX SPACES AND TRANSLATION NUMBERS
}

\author{
ALEKSANDAR POLEKSIĆ \\ (Communicated by Stephen D. Smith)
}

\begin{abstract}
We prove that groups acting geometrically on $\delta$-quasiconvex spaces contain no essential Baumslag-Solitar quotients as subgroups. This implies that they are translation discrete, meaning that the translation numbers of their nontorsion elements are bounded away from zero.
\end{abstract}

The notion of translation numbers is used by many authors, such as J. Alonso and M. Bridson 1], G. Conner [2], S.M. Gersten and H. Short [3] and M. Gromov [5]. If a group is equipped with a word metric, then the translation number of an element in the group may be viewed as the "length" of that element in the group. In "Hyperbolic Groups", Gromov claims that the translation numbers in negatively curved groups are rational with bounded denominator (see also [6]). Conner [2] proves a similar result for $\mathrm{CAT}(0)$ groups, namely that they are translation discrete, meaning that the translation numbers of their nontorsion elements are bounded away from zero. He also gives necessary and sufficient conditions for translation discreteness of quasiconvex groups i.e. groups acting geometrically on quasiconvex spaces. In this paper we prove that these groups, which include all negatively curved and all CAT(0) groups, contain no essential Baumslag-Solitar quotients as subgroups, which in turn implies that they are translation discrete.

Definition. A geodesic triangle in a metric space $X$ is $\delta$-midpoint convex, $\delta \geqslant 0$, if the distance between the midpoints of any two sides of the triangle is no more than half the length of the remaining side plus $\delta$.

Definition. A geodesic metric space $X$ is $\delta$-quasiconvex, if all geodesic triangles in $X$ are $\delta$-midpoint convex.

Using the tripod definition of negatively curved space, one can see that every $\delta$-thin triangle is $2 \delta$-midpoint convex. Therefore, every negatively curved space is quasiconvex. On the other hand, every geodesic triangle in a $\operatorname{CAT}(0)$ space is 0 -midpoint convex and thus every $\operatorname{CAT}(0)$ space is $\delta$-quasiconvex, for every $\delta$.

Received by the editors January 5, 1999

2000 Mathematics Subject Classification. Primary 20F65.

This paper forms a part of the author's Ph.D. dissertation written under the direction of P. Bowers at Florida State University. 
Lemma 1. The distance function on a $\delta$-quasiconvex space $X$ is $4 \delta$-quasiconvex, i.e. for every pair of geodesic paths $c_{1}, c_{2}:[0,1] \mapsto X$ parametrized proportional to arc length and every $t \in[0,1]$,

$$
d\left(c_{1}(t), c_{2}(t)\right) \leqslant(1-t) d\left(c_{1}(0), c_{2}(0)\right)+t d\left(c_{1}(1), c_{2}(1)\right)+4 \delta .
$$

Proof. Define $f:[0,1] \mapsto \mathbb{R}$ by

$$
f(t)=d\left(c_{1}(t), c_{2}(t)\right)-(1-t) d\left(c_{1}(0), c_{2}(0)\right)-t d\left(c_{1}(1), c_{2}(1)\right) .
$$

This is a continuous function on a compact set so it reaches its maximum $f\left(t_{0}\right)=M$, for some $t_{0} \in[0,1]$. Without loss of generality, we may assume that $t_{0} \geqslant 1 / 2$ (otherwise, consider $g(t)=f(1-t)$ ). Let $P$ be the midpoint of a geodesic segment joining $c_{1}\left(2 t_{0}-1\right)$ and $c_{2}(1)$. Then,

$$
\begin{aligned}
f\left(2 t_{0}-1\right) & =d\left(c_{1}\left(2 t_{0}-1\right), c_{2}\left(2 t_{0}-1\right)\right)-\left(2-2 t_{0}\right) d\left(c_{1}(0), c_{2}(0)\right) \\
& -\left(2 t_{0}-1\right) d\left(c_{1}(1), c_{2}(1)\right) \\
& =d\left(c_{1}\left(2 t_{0}-1\right), c_{2}\left(2 t_{0}-1\right)\right)+d\left(c_{1}(1), c_{2}(1)\right) \\
& -2\left[\left(1-t_{0}\right) d\left(c_{1}(0), c_{2}(0)\right)+t_{0} d\left(c_{1}(1), c_{2}(1)\right)\right] \\
& \geqslant 2 d\left(c_{2}\left(t_{0}\right), P\right)-2 \delta+2 d\left(c_{1}\left(t_{0}\right), P\right)-2 \delta-2 d\left(c_{1}\left(t_{0}\right), c_{2}\left(t_{0}\right)\right)+2 M \\
& \geqslant 2 M-4 \delta .
\end{aligned}
$$

Since $f$ attains its maximum at $t_{0}$, it follows that $2 M-4 \delta \leqslant M$, i.e. $M \leqslant 4 \delta$.

From now on, we will think of a geodesic joining $x$ to $y$ as a map $c:[0, \infty) \mapsto X$ such that $\left.c\right|_{[0, d(x, y)]}$ is a geodesic path joining $x$ and $y$ and $c(t)=y$ for $t>d(x, y)$.

Lemma 2. Let $X$ be a $\delta$-quasiconvex space and $c_{1}, c_{2}$ geodesics of finite lengths $l\left(c_{1}\right)$ and $l\left(c_{2}\right)$, respectively. Then, for every $t \geqslant 0$,

$$
d\left(c_{1}(t), c_{2}(t)\right) \leqslant 3 \max \left\{d\left(c_{1}(0), c_{2}(0)\right), d\left(c_{1}\left(l\left(c_{1}\right)\right), c_{2}\left(l\left(c_{2}\right)\right)\right)\right\}+4 \delta .
$$

Proof. Define $c_{i}^{\prime}:[0,1] \mapsto X, i=1,2$, by $c_{i}^{\prime}(t)=c_{i}\left(t l\left(c_{i}\right)\right)$.

If $t \leqslant \min \left\{l\left(c_{1}\right), l\left(c_{2}\right)\right\}$, then

$$
\begin{aligned}
d\left(c_{1}(t), c_{2}(t)\right) & =d\left(c_{1}^{\prime}\left(\frac{t}{l\left(c_{1}\right)}\right), c_{2}^{\prime}\left(\frac{t}{l\left(c_{2}\right)}\right)\right) \\
& \leqslant d\left(c_{1}^{\prime}\left(\frac{t}{l\left(c_{1}\right)}\right), c_{2}^{\prime}\left(\frac{t}{l\left(c_{1}\right)}\right)\right)+d\left(c_{2}^{\prime}\left(\frac{t}{l\left(c_{1}\right)}\right), c_{2}^{\prime}\left(\frac{t}{l\left(c_{2}\right)}\right)\right) \\
& \leqslant \max \left\{d\left(c_{1}(0), c_{2}(0)\right), d\left(c_{1}\left(l\left(c_{1}\right)\right), c_{2}\left(l\left(c_{2}\right)\right)\right)\right\}+4 \delta \\
& +d\left(c_{2}\left(\frac{t l\left(c_{2}\right)}{l\left(c_{1}\right)}\right), c_{2}(t)\right) \\
& \leqslant \max \left\{d\left(c_{1}(0), c_{2}(0)\right), d\left(c_{1}\left(l\left(c_{1}\right)\right), c_{2}\left(l\left(c_{2}\right)\right)\right)\right\}+4 \delta \\
& +\left|l\left(c_{2}\right)-l\left(c_{1}\right)\right| \\
& \leqslant 3 \max \left\{d\left(c_{1}(0), c_{2}(0)\right), d\left(c_{1}\left(l\left(c_{1}\right)\right), c_{2}\left(l\left(c_{2}\right)\right)\right)\right\}+4 \delta .
\end{aligned}
$$


If $l\left(c_{2}\right) \leqslant t<l\left(c_{1}\right)$, then

$$
\begin{aligned}
d\left(c_{1}(t), c_{2}(t)\right) & =d\left(c_{1}^{\prime}\left(\frac{t}{l\left(c_{1}\right)}\right), c_{2}^{\prime}(1)\right) \\
& \leqslant d\left(c_{1}^{\prime}\left(\frac{t}{l\left(c_{1}\right)}\right), c_{2}^{\prime}\left(\frac{t}{l\left(c_{1}\right)}\right)\right)+d\left(c_{2}^{\prime}\left(\frac{t}{l\left(c_{1}\right)}\right), c_{2}^{\prime}(1)\right) \\
& \leqslant \max \left\{d\left(c_{1}(0), c_{2}(0)\right), d\left(c_{1}\left(l\left(c_{1}\right)\right), c_{2}\left(l\left(c_{2}\right)\right)\right)\right\}+4 \delta \\
& +d\left(c_{2}\left(\frac{t l\left(c_{2}\right)}{l\left(c_{1}\right)}\right), c_{2}\left(l\left(c_{2}\right)\right)\right) \\
& =\max \left\{d\left(c_{1}(0), c_{2}(0)\right), d\left(c_{1}\left(l\left(c_{1}\right)\right), c_{2}\left(l\left(c_{2}\right)\right)\right)\right\}+4 \delta \\
& +\frac{l\left(c_{2}\right)\left(l\left(c_{1}\right)-t\right)}{l\left(c_{1}\right)} \\
& \leqslant \max \left\{d\left(c_{1}(0), c_{2}(0)\right), d\left(c_{1}\left(l\left(c_{1}\right)\right), c_{2}\left(l\left(c_{2}\right)\right)\right)\right\}+4 \delta+l\left(c_{1}\right)-t \\
& \leqslant \max \left\{d\left(c_{1}(0), c_{2}(0)\right), d\left(c_{1}\left(l\left(c_{1}\right)\right), c_{2}\left(l\left(c_{2}\right)\right)\right)\right\}+4 \delta+l\left(c_{1}\right)-l\left(c_{2}\right) \\
& \leqslant 3 \max \left\{d\left(c_{1}(0), c_{2}(0)\right), d\left(c_{1}\left(l\left(c_{1}\right)\right), c_{2}\left(l\left(c_{2}\right)\right)\right)\right\}+4 \delta .
\end{aligned}
$$

It is easy to see that the inequality is true for the other choices of $t$.

Definition. A discrete path joining $x$ and $y$ in a metric space $X$ is a map $p$ : $I \mapsto X$ where $I=\left[0, L_{p}\right] \cap \mathbb{N}, L_{p} \in \mathbb{N}$ and $p(0)=x, p\left(L_{p}\right)=y$.

Definition. A discrete path $p$ is called a $(\lambda, \epsilon)$-quasigeodesic, $\lambda \geqslant 1, \epsilon \geqslant 0$, if

$$
\frac{1}{\lambda}\left|t_{1}-t_{2}\right|-\epsilon \leqslant d\left(p\left(t_{1}\right), p\left(t_{2}\right)\right) \leqslant \lambda\left|t_{1}-t_{2}\right|+\epsilon,
$$

for all $t_{1}, t_{2} \in I$.

As for geodesics, we will assume that $p: \mathbb{N} \mapsto X$ by setting $p(t)=p\left(L_{p}\right)$ for $t \geqslant L_{p}$. Here, " $p$ is $(\lambda, \epsilon)$-quasigeodesic" means that $p$ is such when restricted to $\left[0, L_{p}\right] \cap \mathbb{N}$.

Definition. Let $X$ be a metric space and $\mathcal{D}(X)$ the set of all discrete paths in $X$. A quasigeodesic bicombing of $X$ is a section

$$
s: X \times X \mapsto \mathcal{D}(X)
$$

of the endpoints map such that $s_{(x, y)}$ is a $(\lambda, \epsilon)$-quasigeodesic, for all $x, y \in X$.

Definition. A quasigeodesic bicombing is bounded if there are $c_{1} \geqslant 0, c_{2} \geqslant 0$ such that, for all $x_{1}, x_{2}, y_{1}, y_{2} \in X$ and all $t$,

$$
d\left(s_{\left(x_{1}, y_{1}\right)}(t), s_{\left(x_{2}, y_{2}\right)}(t)\right) \leqslant c_{1} \max \left\{d\left(x_{1}, x_{2}\right), d\left(y_{1}, y_{2}\right)\right\}+c_{2} .
$$

Definition. A group $G$ is called semihyperbolic if there is a bounded, quasigeodesic bicombing $s$ of $G$ such that

$$
s_{\left(g h_{1}, g h_{2}\right)}=g s_{\left(h_{1}, h_{2}\right)},
$$

for all $g, h_{1}, h_{2} \in G$.

Definition. Let $G$ be a group with finite generating set $A$ and word metric $d_{A}$. The translation number of the element $g$ of $G$ is

$$
\tau(g)=\lim _{n \rightarrow \infty} \frac{\left|g^{n}\right|}{n},
$$

where $|g|=d_{A}(1, g)$. 
The proof that this limit always exists may be found in 3]. The function $\tau$ has the following properties [3]:

1. $\tau(g)=\tau\left(g^{-1}\right) \leqslant|g|$ for each group element $g$;

2. $\tau$ is a class function, i.e. is constant on conjugacy classes of $G$;

3. $\tau\left(g^{m}\right)=m \tau(g)$ for every group element $g$ and positive integer $m$.

Definition. A group is called translation discrete if there exists $\alpha>0$ such that $\tau(g)>\alpha$, for every nontorsion element $g \in G$.

The following is the result of Alonso and Bridson; see [1].

Theorem 3. If $G$ is a semihyperbolic group, then the translation numbers of nontorsion elements in $G$ are positive.

Definition. Let $G$ be a group of homeomorphisms of $X$. We say that $G$ acts cocompactly on $X$ if there exists a compact set $K \subset X$ such that $G K=X$.

Definition. We say that a group $G$ acting by homeomorphisms on $X$ acts properly discontinously if, for any compact set $K \subset X$,

$$
|\{g \in G \mid g K \cap K \neq \emptyset\}|<\infty .
$$

Definition. A group G acts geometrically on a proper geodesic metric space if it acts by isometries and if its action is cocompact and properly discontinuous.

Theorem 4. If $G$ acts geometrically on a proper $\delta$-quasiconvex space $X$, then $G$ is semihyperbolic.

Proof. Fix $x_{0} \in X$. The map $f: G \mapsto X, f(g)=g x_{0}$ is a quasi-isometry between $G$ and $X$. Therefore, there exist a map $f^{\prime}: X \mapsto G$ and $\lambda, \epsilon \in \mathbb{N}$ such that, for all $g, g_{1}, g_{2} \in G$ and $x, x_{1}, x_{2} \in X$,

1. $d_{X}\left(f\left(g_{1}\right), f\left(g_{2}\right)\right) \leqslant \lambda d_{A}\left(g_{1}, g_{2}\right)+\epsilon$

2. $d_{A}\left(f^{\prime}\left(x_{1}\right), f^{\prime}\left(x_{2}\right)\right) \leqslant \lambda d_{X}\left(x_{1}, x_{2}\right)+\epsilon$;

3. $d_{X}\left(f\left(f^{\prime}(x)\right), x\right) \leqslant \epsilon$;

4. $d_{A}\left(f^{\prime}(f(g)), g\right) \leqslant \epsilon$.

For each $g \in G$ choose a geodesic $r_{g}$ in $X$ joining $x_{0}$ and $g x_{0}$. Define $s_{g}: \mathbb{N} \mapsto G$ by

$$
s_{g}(t)= \begin{cases}1 & \text { if } t=0 \\ f^{\prime}\left(r_{g}(t-1)\right) & \text { if } t \in\left[1, l\left(r_{g}\right)+1\right] \cap \mathbb{N}, \\ g & \text { if } t>l\left(r_{g}\right)+1\end{cases}
$$

Note that the inequality 4 above, together with the fact that $f^{\prime}$ is quasi-isometry, implies that there are $\bar{\lambda} \geqslant 1, \bar{\epsilon} \geqslant 0$ such that, for every $g \in G, s_{g}$ is $(\bar{\lambda}, \bar{\epsilon})$ quasigeodesic. Since $G$ acts by isometries, the bicombing defined by

$$
s_{(g, h)}(t)=g s_{g^{-1} h}(t)
$$

is $(\bar{\lambda}, \bar{\epsilon})$-quasigeodesic. 
To see that it is bounded we proceed as follows: Let $g, h, k, l$ be elements of $G$. Consider the following cases:

1) If $t \in\left[1, l\left(r_{g^{-1} h}\right)+1\right] \cap\left[1, l\left(r_{k^{-1} l}\right)+1\right] \cap \mathbb{N}$, then

$$
\begin{aligned}
d_{A}\left(s_{(g, h)}(t), s_{(k, l)}(t)\right) & =d_{A}\left(g s_{g^{-1} h}(t), k s_{k^{-1} l}(t)\right) \\
& =d_{A}\left(g f^{\prime} r_{g^{-1} h}(t-1), k f^{\prime} r_{k^{-1} l}(t-1)\right) \\
& \leqslant d_{A}\left(g f^{\prime} r_{g^{-1} h}(t-1), f^{\prime} f g f^{\prime} r_{g^{-1} h}(t-1)\right) \\
& +d_{A}\left(f^{\prime} f g f^{\prime} r_{g^{-1} h}(t-1), f^{\prime} f k f^{\prime} r_{k^{-1} l}(t-1)\right) \\
& +d_{A}\left(f^{\prime} f k f^{\prime} r_{k^{-1} l}(t-1), k f^{\prime} r_{k^{-1} l}(t-1)\right) \\
& \leqslant \lambda d_{X}\left(f g f^{\prime} r_{g^{-1} h}(t-1), f k f^{\prime} r_{k^{-1} l}(t-1)\right)+3 \epsilon \\
& =\lambda d_{X}\left(g f f^{\prime} r_{g^{-1} h}(t-1), k f f^{\prime} r_{k^{-1} l}(t-1)\right)+3 \epsilon \\
& \leqslant \lambda\left[d_{X}\left(g f f^{\prime} r_{g^{-1} h}(t-1), g r_{g^{-1} h}(t-1)\right)\right. \\
& +d_{X}\left(g r_{g^{-1} h}(t-1), k r_{k^{-1} l}(t-1)\right) \\
& \left.+d_{X}\left(k r_{k^{-1} l}(t-1), k f f^{\prime} r_{k^{-1} l}(t-1)\right)\right]+3 \epsilon \\
& \leqslant \lambda d_{X}\left(g r_{g^{-1} h}(t-1), k r_{k^{-1} l}(t-1)\right)+(2 \lambda+3) \epsilon \\
& \leqslant \lambda\left[3 \max _{x}\left\{d_{X}\left(g x_{0}, k x_{0}\right), d_{X}\left(h x_{0}, l x_{0}\right)\right\}+4 \delta\right]+(2 \lambda+3) \epsilon \\
& \leqslant 3 \lambda \max \left\{\lambda d_{A}(g, k)+\epsilon, \lambda d_{A}(h, l)+\epsilon\right\}+4 \delta \lambda+(2 \lambda+3) \epsilon \\
& =3 \lambda^{2} \max \left\{d_{A}(g, k), d_{A}(h, l)\right\}+4 \delta \lambda+(5 \lambda+3) \epsilon .
\end{aligned}
$$

The third inequality from the bottom follows from Lemma 2

2) Cases $t=0$ and $t>\max \left\{l\left(r_{g^{-1} h}\right)+1, l\left(r_{k^{-1} l}\right)+1\right\}$ are easy.

To finish with the proof, it is enough to consider one more case:

3) If $l\left(r_{g^{-1} h}\right)+1<t \leqslant l\left(r_{k^{-1} l}\right)+1$, then

$$
\begin{aligned}
d_{A}\left(s_{(g, h)}(t), s_{(k, l)}(t)\right) & =d_{A}\left(g s_{g^{-1} h}(t), k s_{k^{-1} l}(t)\right) \\
& =d_{A}\left(g g^{-1} h, k s_{k^{-1} l}(t)\right) \\
& \leqslant d_{A}\left(g g^{-1} h, g f^{\prime}\left(r_{g^{-1} h}(t-1)\right)\right) \\
& +d_{A}\left(g f^{\prime} r_{g^{-1} h}(t-1), k f^{\prime} r_{k^{-1} l}(t-1)\right) \\
& \leqslant 3 \lambda^{2} \max \left\{d_{A}(g, k), d_{A}(h, l)\right\}+4 \delta \lambda+(5 \lambda+4) \epsilon .
\end{aligned}
$$

The last inequality follows from 1).

Corollary 5. If $G$ acts geometrically on a proper $\delta$-quasiconvex metric space $X$, then $G$ contains no essential Baumslag-Solitar quotient i.e. quotient group of

$$
B_{n, m}=\left\langle a, b \mid a^{-1} b^{m} a=b^{n}\right\rangle, m>n>0,
$$

as a subgroup.

Proof. Observe that a group contains an essential B-S quotient if and only if it contains an element of infinite order having two different conjugate powers. Apply properties 2 and 3 of translation function and Theorems 3 and 4 .

The following two are Conner's results [2], modified for the setting of the word metric. 
Theorem 6. If $G$ is a group of isometries of a proper, $\delta$-quasiconvex space $X$, then for all $g \in G$,

$$
\tau(g) \geqslant \frac{1}{\lambda}\left(d_{0}(g)-2 \delta\right),
$$

where $\lambda$ is the quasi-isometry constant from the proof of Theorem 4 and $d_{0}(g)=$ $\inf \left\{d_{X}(x, g x) \mid x \in X\right\}$.

Proof. Let $x \in X$. Let $s$ be a geodesic segment joining $x$ and $g x$ and let $r$ be a geodesic segment joining $x$ and $g^{2} x$. Let $p, q$ be the midpoints of $s$ and $g s$ respectively. Observe that $q=g p$. Since $(s, g s, r)$ is $\delta$-midpoint convex, we have

Hence

$$
d_{0}(g) \leqslant d(p, q) \leqslant \frac{1}{2} d\left(x, g^{2} x\right)+\delta .
$$

$$
d\left(x, g^{2} x\right) \geqslant 2\left(d_{0}(g)-\delta\right) .
$$

Since $x$ was arbitrary,

$$
d_{0}\left(g^{2}\right) \geqslant 2\left(d_{0}(g)-\delta\right) .
$$

Since $g$ was arbitrary, by induction,

$$
d_{0}\left(g^{2^{n}}\right) \geqslant 2^{n}\left[d_{0}(g)-\left(1+\frac{1}{2}+\cdots+\frac{1}{2^{n-1}}\right) \delta\right] .
$$

In the setting of the proof of Theorem 4.

$$
\lambda d_{A}\left(1, g^{2^{n}}\right)+\epsilon \geqslant d_{X}\left(x_{0}, g^{2^{n}} x_{0}\right) \geqslant d_{0}\left(g^{2^{n}}\right) .
$$

It follows that

and thus,

$$
d_{A}\left(1, g^{2^{n}}\right) \geqslant \frac{1}{\lambda}\left\{2^{n}\left[d_{0}(g)-\left(1+\frac{1}{2}+\cdots+\frac{1}{2^{n-1}}\right) \delta\right]-\epsilon\right\},
$$

$$
\tau(g) \geqslant \frac{1}{\lambda}\left(d_{0}(g)-2 \delta\right)
$$

Observation: Note that $d_{0}$ is a class function, i.e. it is constant on conjugacy classes of $G$.

Theorem 7. If $G$ acts geometrically on a proper $\delta$-quasiconvex metric space $X$, then, for every $\alpha>0$, there are only finitely many conjugacy classes $C$ with $\tau(C)<$ $\alpha$.

Proof. Let $K$ be a compact set such that $G K=X$. Fix $x_{0} \in X$. Let $r>0$ be such that $\overline{B_{r}}\left(x_{0}\right) \supseteq K$. Let $C$ be a conjugacy class of $G$ such that $d_{0}(C)<\beta$, where $\beta=2 \delta+\lambda \alpha$, and $\lambda$ as in the previous theorem. Choose $\gamma \in C$ and $x \in K$ so that $d_{X}(x, \gamma x)<\beta$. Then $\gamma x \in \overline{\gamma B_{r+\beta}}\left(x_{0}\right) \cap \overline{B_{r+\beta}}\left(x_{0}\right)$. Since the action of $G$ is properly discontinuous, i.e. $\left\{\gamma \mid \gamma \overline{B_{r+\beta}}\left(x_{0}\right) \cap \overline{B_{r+\beta}}\left(x_{0}\right) \neq \emptyset\right\}$ is finite, it follows that there are only finitely many such conjugacy classes $C$. The result now follows from Theorem 6 .

Theorem 8. If $G$ acts geometrically on a proper $\delta$-quasiconvex metric space $X$, then $G$ is translation discrete.

Proof. Follows from Theorems 3 4 and 7. 
Corollary 9. If $G$ acts geometrically on a proper $\delta$-quasiconvex metric space, then the translation numbers of elements in $G$ form a discrete subset of nonnegative reals.

\section{REFERENCES}

[1] J. Alonso, M. Bridson, Semihyperbolic groups, Proc. LMS (3) 70 (1995), 56-114. MR 95j:20033

[2] G. Conner, Translation numbers of groups acting on convex spaces, Brigham Young University, preprint (1994).

[3] S.M. Gersten, H. Short, Rational subgroups of biautomatic groups, Annals of Math. 134 (1991), 125-158. MR 92g:20092

[4] E. Ghys and P. de la Harpe, Infinite groups as geometric objects (after Gromov), Chapter 10 of Ergodic Theory, Symbolic Dynamics, and Hyperbolic Spaces (T. Bedford, M. Keane, and C. Series, Eds.), Oxford Univ. Press (1991), 299-314. CMP 92:02

[5] M. Gromov, Hyperbolic groups, in Essays in Group Theory (S.M. Gersten, ed., Springer-Verlag, 1987), 75-263. MR 89e:20070

[6] A. Poleksic, Translation numbers in negatively curved groups, Topol. and its Appl., to appear.

[7] A. Poleksic, The boundary of a quasiconvex space, Topol. and its Appl., to appear.

Cold Spring Harbor Laboratory, P.O. Box 100, 1 Bungtown Road, Cold Spring HarBOR, NEW YORK 11724

E-mail address: poleksic@cshl.org 